Voix et Images

voixetimages

\title{
Bibliographie de Louis Hamelin
}

\section{Laurence Perron}

Volume 41, numéro 1 (121), automne 2015

Louis Hamelin

URI : https://id.erudit.org/iderudit/1033962ar

DOI : https://doi.org/10.7202/1033962ar

Aller au sommaire du numéro

Éditeur(s)

Université du Québec à Montréal

ISSN

0318-9201 (imprimé)

1705-933X (numérique)

Découvrir la revue

Citer ce document

Perron, L. (2015). Bibliographie de Louis Hamelin. Voix et Images, 41(1),

101-119. https://doi.org/10.7202/1033962ar

Ce document est protégé par la loi sur le droit d'auteur. L'utilisation des services d'Érudit (y compris la reproduction) est assujettie à sa politique d'utilisation que vous pouvez consulter en ligne.

https://apropos.erudit.org/fr/usagers/politique-dutilisation/
Cet article est diffusé et préservé par Érudit.

Érudit est un consortium interuniversitaire sans but lucratif composé de l’Université de Montréal, l'Université Laval et l'Université du Québec à Montréal. Il a pour mission la promotion et la valorisation de la recherche. https://www.erudit.org/fr/ 


\section{B I B LIOGRA PHIE DE LOUIS HAMELIN}

$+++$

\section{LAURENCE PERRON}

Université du Québec à Montréal

\section{E U V R E S}

\section{A. Romans et recueils de nouvelles}

+ La rage, Montréal, Québec Amérique, coll. «Littérature d’Amérique», 1989, 405 p. (Rééditions: Montréal, TYPO, 1995, 516 p.; Montréal, Boréal, coll. «Boréal compact», 2010, 568 p.)

+ Ces spectres agités, Montréal, XYZ éditeur, coll. «Romanichels», 1991, 282 p. (Rééditions: Montréal, TYPO, 1993, 293 p; Montréal, XYZ éditeur, 2003, 288 p; Montréal, Boréal, coll. «Boréal compact», 2010, 312 p.)

+ Cowboy, Montréal, XYZ éditeur, coll. «Romanichels», 1992, 417 p. (Rééditions: Montréal, XYZ éditeur, coll. «Romanichels», 1998, 440 p; Montréal, Boréal, coll. «Boréal compact», 2009, 480 p.)

+ Betsi Larousse ou l'ineffable eccéité de la loutre, Montréal, XYZ éditeur, coll. «Romanichels», 1994, 270 p. (Réédition: Montréal, Boréal, coll. «Boréal compact», 2009, 311 p.)

+ Le soleil des gouffres, Montréal, Boréal, 1996, 372 p. (Réédition: Paris, Stock, 1997, 372 p.)

+ Le joueur de flûte, Montréal, Boréal, 2001, 228 p. (Réédition: Montréal, Boréal, coll. «Boréal compact», 2006, 228 p.)

+ Sauvages, Montréal, Boréal, 2006, 289 p. (Réédition: Montréal, Boréal, coll. «Boréal compact», 2013, 289 p.)

+ La constellation du Lynx, Montréal, Boréal, 2010, 600 p. (Réédition: Montréal, Boréal, coll. «Boréal compact», 2012, 600 p.)

\section{B. Fe uille ton}

+ Les étranges et édifiantes aventures d'un oniromane, Québec, L'instant même, 1994, 72 p.

\section{Mémoire de maîtrise}

+ Ces spectres agités suivi d'Entre la parodie et le vrai: la création comme figure d'interférence, Montréal, Université du Québec à Montréal, 1990, 315 f.

D. S cé n a rio

+ Yves Sioui Durand (réal.), Mesnak, K-Films Amérique, 2012, 96 min.

\section{E. E s s a is}

+ L’humain isolé, Trois-Pistoles, Éditions Trois-Pistoles, 2006, 176 p. 
+ Fabrications. Essai sur la fiction et l'histoire, Montréal, Presses de l'Université de Montréal, coll. «Prix de la revue Études françaises», 2014, 232 p.

\section{F. Textes littéraires parus dans des périodiques et des} collectifs

+ «Le sourire du cordonnier», Ciel variable, $\mathrm{n}^{\circ} 11$, printemps 1990, p. 32-33.

+ «Le voyage à Pandémonium», XYZ. La revue de la nouvelle, n 25 , printemps-février 1991, p. 31-40.

+ «Sans amour ni trompette», XYZ. La revue de la nouvelle, n 28, 1991, p. 38.

+ «Ce que je ferai quand je serai grand», Mœbius, n 50, automne 1991, p. 51-58.

+ «Le jour où le grand Larry clamsa», Stanley Péan (dir.), Complicités, Montréal, PAJE Éditeur/ Revue Stop, 1991, p. 67-83.

+ «J'ai vu un ectoplasme», Liberté, vol. 34, n² 2, avril 1992, p. 37-38.

+ «La rouge et la noire», Stanley Péan (dir.), Évasion, Montréal, Revue Stop, 1992, p. 67-75.

+ «Portrait de Ti-Luc Blouin», Stop, no 131, 1993, p. 63-72.

+ «L'autour», Gilles Pellerin (dir.), Circonstances particulières, Québec, L'instant même, 1998, p. 135-147.

+ «L'inénarrable ubiquité de l'écureuil», Liberté, vol. 48, n 2, mai 2006, p. 18-19.

+ Avec Domingo Cisneros, «Le chant du pure-fourrure», Laure Morali (dir.), Aimititau! Parlonsnous!, Montréal, Mémoire d'encrier, 2008, p. 61-83.

+ «La tentation idyllique», La pratique du roman, Isabelle Daunais et François Ricard (dir.), Montréal, Boréal, 2012, p. 25-41.

+ «Deal», Nouveau Projet, n 4, automne-hiver 2013, p. 121-126.

+ «The Price is Right», Les écrits, nº 137, mars 2013, p. 11-47.

\section{G. Avant-propos, textes d'opinion et communications}

+ Le voyage en pot. Chroniques 1998-1999, Montréal, Boréal, coll. «Papiers collés», 1999, 234 p.

\section{H. Articles parus dans des quotidiens}

+ «Nous assisterons au retour des stylistes: les années 90 - Littérature», La Presse, 6 janvier 1990, p. K3.

+ «Éloge complaisant du Pinochétisme», Le Devoir, 29 septembre 1993, p. A7.

+ «D Rémi Fassol, phumiste», Le Devoir, 25 juin 1994, p. A1.

+ «L'auteur de Oka indigne», Le Devoir, 2 juillet 1994, p. A1.

+ «La motivation», Le Devoir, 9 juillet 1994, p. A1.

+ «L'empire de Trébizonde», Le Devoir, 16 juillet 1994, p. A1.

+ «Les racines de l'enfer», Le Devoir, 23 juillet 1994, p. A1.

+ «Les onagres de l'ONU», Le Devoir, 30 juillet 1994, p. A1.

+ «Montréal - Québec - Canada», Ici, vol. 1, nº 16, 8-15 janvier 1998, p. 14.

+ «Début de l'odyssée parisienne», Ici, vol. 1, no 17, 15-22 janvier 1998, p. 16.

+ «Louis Hamelin s'initie aux joies de son studio parisien », Ici, vol. 1, n 18, 22 -29 janvier 1998, p. 16.

+ «Retour sur les patriotes», Ici, vol. 1, nº 19, 29 janvier - 5 février 1998, p. 14.

+ «Le spleen vu de Paris», Ici, vol. 1, n 20, 5-12 février 1998, p. 20.

+ «Sur invitation», Ici, vol. 1, nº 21, 12-19 février 1998, p. 18. 
+ «Le vol magique», Ici, vol. 1, n 22, 21-28 février 1998, p. 18.

+ «Requiem pour Ti-Corps», Ici, vol. 1, n 23, 28 février-5 mars 1998, p. 16.

+ «Le cavalier et l'Indien», Ici, vol. 1, n 24, 5-12 mars 1998, p. 10.

+ "Allô toi», Ici, vol. 1, n 25, 12-19 mars 1998, p. 10.

+ «Cœur-de-Gerfaut», Ici, vol. 1, n² 26, 19-26 mars 1998, p. 10.

+ «Ceux qui nous aiment», Ici, vol. 1, n 27, 26 mars-2 avril 1998, p. 8.

+ «La connerie littéraire», Ici, vol. 1, n 28, 2-9 avril 1998, p. 10.

+ «Probablement l'Espagne», Ici, vol. 1, n 30, 16-23 avril 1998, p. 10.

+ «Ma guerre d'Espagne», Ici, vol. 1, n 31, 23-30 avril 1998, p. 10.

+ «Le travail c'est la liberté», Ici, vol. 1, no 33, 7-14 mai 1998, p. 7.

+ «Rêve gitan», Ici, vol. 1, n 34, 14-21 mai 1998, p. 9.

+ "Barreaux», Ici, vol. 1, n 35, 21-28 mai 1998, p. 7.

+ «Les babouins et les oies», Ici, vol. 1, n 36, 28 mai-4 juin 1998, p. 7.

+ "Souvenir», Ici, vol. 1, n 37, 4-11 juin 1998, p. 8.

+ «La France», Ici, vol. 1, n 38, 11-18 juin 1998, p. 8.

+ «Pied de guerre», Ici, vol. 1, n 39, 18-25 juin 1998, p. 8 .

+ "Le temps», Ici, vol. 1, n 40, 25 juin-2 juillet 1998, p. 6.

+ «La France (suite et fin)», Ici, vol. 1, n 41, 2-9 juillet 1998, p. 8.

+ «Hymnes», Ici, vol. 1, nº 42, 9-16 juillet 1998, p. 8.

+ «Sang bleu ou le feu d'artifice», Ici, vol. 1, n 43, 16-21 juillet 1998, p. 8.

+ «Lettres à L.A. Rodriguez», Ici, vol. 1, n 45, 3 juillet-6 août 1998, p. 8.

+ "Les élus», Ici, vol. 1, n 46, 6-13 août 1998, p. 10.

+ «Les sources», Ici, vol. 1, n 47, 13-20 août 1998, p. 8.

+ «Voir», Ici, vol. 1, n 50, 3-10 septembre 1998, p. 8.

+ «Le révolutionnaire et la tortue», Ici, vol. 1, n 51, 10-17 septembre 1998, p. 10.

+ «Le révolutionnaire et la tortue (2)», Ici, vol. 1, n 52, 17-24 septembre 1998, p. 9.

+ «Le gros Y», Ici, vol. 2, nº 1, 24 septembre- ${ }^{\text {er }}$ octobre 1998, p. 8.

+ «Folies», Ici, vol. 2, nº 2, 1 er-8 octobre 1998, p. 6.

+ «Le goût de la chasse», Ici, vol. 2, n 3, 8-15 octobre 1998, p. 10.

+ "Ahimsa ou l'Amérique de rêve», Le Devoir, 10 juillet 1999, p. D4.

+ «Vrai ou faux», Le Devoir, 31 juillet 1999, p. D5.

+ «Miséricorde», Le Devoir, 14 août 1999, p. D5.

+ «Un cas possible de justice poétique» Le Devoir, 21 août 1999, p. D5.

+ «Romans américains: de l'Irlande à Pékin», Le Devoir, 28 août 1999, p. D8.

+ «Génétique de la passion», Le Devoir, 4 septembre 1999, p. D4.

+ «L'ombre de Faulkner», Le Devoir, 23 octobre 1999, p. D4.

+ «Papa a raison», Le Devoir, 6 novembre 1999, p. D4.

+ «Romans d'Amérique», Le Devoir, 13 novembre 1999, p. D14.

+ «Un bon plombier», Le Devoir, 27 novembre 1999, p. D6.

+ «La nuit du président», Le Devoir, 11 décembre 1999, p. D6.

+ «Des nouvelles du New Hampshire», Le Devoir, 18 décembre 1999, p. D6.

+ «La trempe des bâtisseurs», Le Devoir, 24 décembre 1999, p. D5.

+ «La nature du kitsch», Le Devoir, 31 décembre 1999, p. B5.

+ «Où est la vie?», Le Devoir, 22 janvier 2000, p. D5.

+ «Romans de l'Amérique», Le Devoir, 29 janvier 2000, p. D5. 
+ «Viande à poil», Le Devoir, 5 février 2000, p. D4.

+ «Pardonnez-lui, seigneur...», Le Devoir, 12 février 2000, p. D4.

+ "Mailer ou l'Amérique», Le Devoir, 19 février 2000, p. D6.

+ "Ce que je veux quand je dis cela», Le Devoir, 4 mars 2000, p. D5.

+ "Le degré zéro du désir», Le Devoir, 11 mars 2000, p. D6.

+ «Un besoin en nourrit un autre», Le Devoir, 18 mars 2000, p. D8.

+ «L'escouade de la moralité», Le Devoir, $1^{\mathrm{er}}$ avril 2000, p. D7.

+ "Mots d'amour», Le Devoir, 8 avril 2000, p. D8.

+ «Le temps des Indiens», Le Devoir, 8 avril 2000, p. D13.

+ «Le retour de l'homme invisible», Le Devoir, 15 avril 2000, p. D5.

+ «L'honneur des petites filles perdues», Le Devoir, 6 mai 2000, p. D4.

+ «Passion ruminée», Le Devoir, 13 mai 2000, p. D4.

+ «Les contes de la zappette», Le Devoir, 27 mai 2000, p. D5.

+ «Drapeau rouge et liste noire», Le Devoir, 10 juin 2000, p. D5.

+ «L'intelligence du vide», Le Devoir, 17 juin 2000, p. D5.

+ «Jim Harrisson est les mâchoires de la vie», Le Devoir, 24 juin 2000, p. D5.

+ «Responsables...», Le Devoir, 15 juillet 2000, p. D6.

+ «La moindre lueur», Le Devoir, 22 juillet 2000, p. D6.

+ «Sur la route des Hell's Angels», Le Devoir, 29 juillet 2000, p. D5.

+ "Mort d'une bonne bourgeoise», Le Devoir, 19 août 2000, p. D4.

+ «L'École du flegme», Le Devoir, 9 septembre 2000, p. D6.

+ "Mondes possibles», Le Devoir, 23 septembre 2000, p. D5.

+ «Nous sommes de la mémoire», Le Devoir, 4 novembre 2000, p. D5.

+ «Canaméricain», Le Devoir, 18 novembre 2000, p. D8.

+ «Juste bonne», Le Devoir, 2 décembre 2000, p. D6.

+ «Photographie», Le Devoir, 9 décembre 2000, p. D14.

+ «L'évidence brute», Le Devoir, 16 décembre 2000, p. D11.

+ «Voie de dépassement», Le Devoir, 30 décembre 2000, p. D5.

+ «Menaces», Le Devoir, 13 janvier 2001, p. D5.

+ «Écrire sous la pluie», Le Devoir, 24 mars 2001, p. D5.

+ «Danger privé», Le Devoir, 7 avril 2001, p. D11.

+ «Les ravages de l'ennui», Le Devoir, 5 mai 2001, p. D5.

+ "Adieu, les vrais», Le Devoir, 8 septembre 2001, p. D6.

+ «Salut de John Fante», Le Devoir, 22 septembre 2001, p. D6.

+ "L'île au désir», Le Devoir, 20 octobre 2001, p. D10.

+ "L'adieu à l'Irlande», Le Devoir, $1^{\text {er }}$ décembre 2001, p. D6.

+ «Le fonds de commerce», Le Devoir, 29 décembre 2001, p. D6.

+ «Un chant païen», Le Devoir, 12 janvier 2002, p. D5.

+ «Macroescroquerie», Le Devoir, 2 février 2002, p. D6.

+ «Les années de crainte», Le Devoir, 9 mars 2002, p. D5.

+ "Du côté de chez Fred», Le Devoir, 23 mars 2002, p. D5.

+ «Comme chez les Grecs... », Le Devoir, 13 avril 2002, p. D5.

+ «Les blés», Le Devoir, 26 juillet 2003, p. E1.

+ "Cent ans de sollicitude», Le Devoir, 11 octobre 2003, p. F1.

+ «Roman québécois: Vichy au régime», Le Devoir, 18 octobre 2003, p. F1. 
+ «Donneur de sens», Le Devoir, $1^{\mathrm{er}}$ novembre 2003, p. F6.

+ «La planète aux voix», Le Devoir, 15 novembre 2003, p. F8.

+ "Du cachot au château ", Le Devoir, 6 décembre 2003, p. F8.

+ «Problèmes de français», Le Devoir, 7 février 2004, p. F4.

+ «Rosebud», Le Devoir, 7 février 2004, p. A7.

+ «La frontière de Rushdie», Le Devoir, 14 février 2004, p. F1.

+ «Le pays de Sam», Le Devoir, 20 mars 2004, p. F9.

+ «Ça me botte», Le Devoir, 27 mars 2004, p. F4.

+ "Le "siège périlleux" de Richler», Le Devoir, 3 avril 2004, p. F6.

+ "Choses vues", Le Devoir, 10 avril 2004, p. F9.

+ «Les royaumes de Gilles Pellerin», Le Devoir, 17 avril 2004, p. F5.

+ «Essence américaine», Le Devoir, 24 avril 2004, p. F4.

+ «Les secrets de la licorne», Le Devoir, $1^{\text {er }}$ mai 2004, p. F6.

+ «La guerre? Non madame...», Le Devoir, 8 mai 2004, p. F4.

+ «L'amour-volcan», Le Devoir, 15 mai 2004, p. F4.

+ «Lectures d'été - Misère de misère», Le Devoir, 12 juin 2004, p. F5.

+ «Les chiens savants», Le Devoir, 24 juillet 2004, p. E1.

+ «Pour l'amour, j'ai une fille à Munich», Le Devoir, 4 septembre 2004, p. F4.

+ «L'histoire secrète», Le Devoir, 2 octobre 2004, p. F4.

+ "Là-bas dans la forêt», Le Devoir, 30 octobre 2004, p. F6.

+ «Elizabeth en spectacle», Le Devoir, 6 novembre 2004, p. F6.

+ «Le bonheur ardu d'écrire», Le Devoir, 13 novembre 2004, p. F10.

+ "Un grand roman épique signé Tomson Highway», Le Devoir, 4 décembre 2004, p. F4.

+ «Mélanie au nom de fleuve», Le Devoir, 18 décembre 2004, p. F6.

+ «Les histoires des autres», Le Devoir, 8 janvier 2005, p. E2.

+ «La vie», Le Devoir, 26 février 2005, p. F4.

+ «L'explorateur en kilt», Le Devoir, 2 avril 2005, p. F4.

+ "L'explication des braises», Le Devoir, 9 avril 2005, p. F5.

+ «Du côté de chez Sean», Le Devoir, 30 avril 2005, p. F4.

+ «Un revenant comme un autre», Le Devoir, 21 mai 2005, p. F4.

+ «Les malheureux naufragés», Le Devoir, 28 mai 2005, p. F5.

+ «Vue du pont, vue de l'enfance», Le Devoir, 11 juin 2005, p. F4.

+ «Parfaitement raté», Le Devoir, 25 juin 2005, p. E6.

+ «Il restera toujours le Wyoming», Le Devoir, 16 juillet 2005, p. E2.

+ «Du béton», Le Devoir, 23 juillet 2005, p. E5.

+ "Guérillas», Le Devoir, 3 septembre 2005, p. F4.

+ «Entre deux boucheries», Le Devoir, 10 septembre 2005, p. F4.

+ "Pas la chasse aux papillons», Le Devoir, 8 octobre 2005, p. F5.

+ «Docteur Kinsey et Mister Prok», Le Devoir, 29 octobre 2005, p. F4.

+ «L'homme qui s'empêcha de vivre», Le Devoir, 5 novembre 2005, p. F5.

+ «Le dieu du feu», Le Devoir, 12 novembre 2005, p. F17.

+ «Jouir», Le Devoir, 19 novembre 2005, p. F6.

+ «Écrire à la cuiller», Le Devoir, 26 novembre 2005, p. F5.

+ «Le soleil se lève aussi à l'ouest», Le Devoir, 14 janvier 2006, p. E12.

+ «Mordecai dans tous ses états», Le Devoir, 21 janvier 2006, p. F4. 
+ "Cinquante terrains de football par jour», Le Devoir, 4 février 2006, p. F4.

+ «Voyage au Portugal avec deux inspecteurs de police», Le Devoir, 18 mars 2006, p. F4.

+ «Les mots qui nous font», Le Devoir, 25 mars 2006, p. F4.

+ "L'école des cadavres», Le Devoir, $1^{\text {er }}$ avril 2006, p. F5.

+ «Le retour de Chien Brun», Le Devoir, 15 avril 2006, p. F6.

+ «Je suis de l'eau», Le Devoir, 29 avril 2006, p. F4.

+ "Quelque part entre le backstop et Broadway...», Le Devoir, 6 mai 2006, p. F6.

+ «Le blues de l'abattoir», Le Devoir, 20 mai 2006, p. F4.

+ «Il était une fois dans l'Ouest canadien», Le Devoir, 27 mai 2006, p. F5.

+ «Le cas de l'oncle Tom», Le Devoir, 3 juin 2006, p. F5.

+ "L'été pour ça», Le Devoir, 16 juin 2006, p. F4.

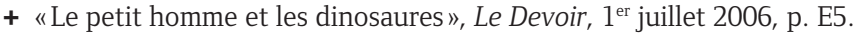

+ «La chair humaine», Le Devoir, 22 juillet 2006, p. E5.

+ «Le peuple des écritures», Le Devoir, 29 juillet 2006, p. E6.

+ «Littérature extrême», Le Devoir, 5 août 2006, p. E2.

+ «D'Amours, P.Q.», Le Devoir, 12 août 2006, p. E2.

+ «Le saumon», Le Devoir, 19 août 2006, p. E2.

+ «Entre voisins», Le Devoir, 2 septembre 2006, p. F4.

+ «La mauvaise graine», Le Devoir, 9 septembre 2006, p. F5.

+ "La beauté du monde», Le Devoir, 16 septembre 2006, p. F7.

+ «De Shakespeare à Carver», Le Devoir, 23 septembre 2006, p. F5.

+ "Ameriquois», Le Devoir, 7 octobre 2006, p. F5.

+ "Ville-cancer pour un livre-tumeur», Le Devoir, 14 octobre 2006, p. F5.

+ «Dans le rôle de la victime», Le Devoir, 21 octobre 2006, p. F6.

+ «Habiter la bite», Le Devoir, 11 novembre 2006, p. F6.

+ "Comment devenir un héros», Le Devoir, 18 novembre 2006, p. A1.

+ «La guerre, yes mon colonel», Le Devoir, 20 novembre 2006, p. A1.

+ «La rue qui perdit son nom», Le Devoir, 21 novembre 2006, p. A1.

+ «Comme au cinéma», Le Devoir, 9 décembre 2006, p. F6.

+ «José Acquelin et la lune», Le Devoir, 16 décembre 2006, p. F4.

+ "Les motels et les choses», Le Devoir, 6 janvier 2007, p. E7.

+ «Mémoires américaines», Le Devoir, 20 janvier 2007, p. F4.

+ «Un temps de verbe plus tard dans les Maritimes», Le Devoir, 3 février 2007, p. F4.

+ «Il y aura toujours le Montana», Le Devoir, 10 février 2007, p. F5.

+ "Prendre un lecteur par la main», Le Devoir, 17 février 2007, p. F6.

+ «Le spectacle de la mort», Le Devoir, 3 mars 2007, p. F5.

+ «Génial, hub», Le Devoir, 10 mars 2007, p. F3.

+ «La culture cultivée», Le Devoir, 24 mars 2007, p. F4.

+ «L'homme est un loup», Le Devoir, 31 mars 2007, p. F4.

+ «En direct de l'Interzone», Le Devoir, 7 avril 2007, p. F6.

+ «Mon nom est Légions», Le Devoir, 14 avril 2007, p. F4.

+ «Kurt Vonnegut est tombé sur la tête», Le Devoir, 21 avril 2007, p. F5.

+ "Courez toujours», Le Devoir, 28 avril 2007, p. F4.

+ «Incroyablement indicible et terriblement bavard», Le Devoir, 12 mai 2007, p. F5.

+ «Comment lire Louise Erdrich et les autres», Le Devoir, 19 mai 2007, p. F5. 
+ «Le chanteur et le général», Le Devoir, 26 mai 2007, p. F4.

+ «Salinger vivant», Le Devoir, 2 juin 2007, p. F4.

+ «Une prison appelée Liberté», Le Devoir, 9 juin 2007, p. F4.

+ «Jim Harrison incarné», Le Devoir, 16 juin 2007, p. F5.

+ «Département de la fiction», Le Devoir, 23 juin 2007, p. E8.

+ "Où était passé Charles», Le Devoir, 30 juin 2007, p. E2.

+ «Littérature américaine - Les pieds dans la bouse», Le Devoir, 7 juillet 2007, p. E7.

+ «La chevauchée de Babel», Le Devoir, 14 juillet 2007, p. E2.

+ «Le roman d'espionnage», Le Devoir, 21 juillet 2007, p. E7.

+ «Homos on the range», Le Devoir, 28 juillet 2007, p. E3.

+ «Le paradis des demoiselles», Le Devoir, 4 août 2007, p. E8.

+ «Il était guerrier», Le Devoir, 18 août 2007, p. E7.

+ «Les maudits», Le Devoir, 15 septembre 2007, p. F4.

+ "Du côté de la "shed"», Le Devoir, 22 septembre 2007, p. F5.

+ «J'ai épousé un socialiste», Le Devoir, 29 septembre 2007, p. F4.

+ «Giuseppe Bonaviri, ses oiseaux et ses guerres», Le Devoir, 13 octobre 2007, p. F4.

+ "L'usine à cadavres», Le Devoir, 20 octobre 2007, p. F4.

+ «La mémoire des armes», Le Devoir, 27 octobre 2007, p. F4.

+ "Gérin-Lajoie, premier violon», Le Devoir, 3 novembre 2007, p. F4.

+ «Les nuls et les morts», Le Devoir, 17 novembre 2007, p. F5.

+ «Les neiges du Kazakhstan», Le Devoir, 24 novembre 2007, p. F4.

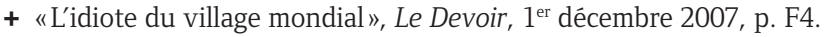

+ «Le mal-pensant», Le Devoir, 8 décembre 2007, p. F4.

+ «Le point de vue du mur», Le Devoir, 15 décembre 2007, p. F5.

+ «Il était une fois...», Le Devoir, 22 décembre 2007, p. F4.

+ "Guerre et paix sur la terre aux hommes de bonne volonté», Le Devoir, 29 décembre 2007, p. E5.

+ "La reine des bécosses», Le Devoir, 5 janvier 2008, p. E2.

+ «Les joueurs de piano», Le Devoir, 12 janvier 2008, p. F4.

+ «Plus rien ne m’étonne», Le Devoir, 19 janvier 2008, p. F4.

+ «Les fils d'Abraham», Le Devoir, 26 janvier 2008, p. F4.

+ «Les 3 p'tits éditeurs...», Le Devoir, 2 février 2008, p. F4.

+ «Le bout de la route», Le Devoir, 9 février 2008, p. F4.

+ «Les diamants ne sont pas éternels», Le Devoir, 16 février 2008, p. F4.

+ «Les mots de la fin», Le Devoir, 23 février 2008, p. F4.

+ «L'Amérique-Monde», Le Devoir, $1^{\text {er }}$ mars 2008, p. F4.

+ «Tant de mots pour une seule chose triste», Le Devoir, 8 mars 2008, p. F4.

+ "Le calme incandescent de l'authentique possédé», Le Devoir, 15 mars 2008, p. F4.

+ «Promesses d'ivrogne», Le Devoir, 22 mars 2008, p. F4.

+ «Contre l'école militaire», Le Devoir, 29 mars 2008, p. F4.

+ «La littérature est l'histoire d'une vieille dame», Le Devoir, 5 avril 2008, p. F4.

+ «La déclaration de Pointe-Bleue», Le Devoir, 12 avril 2008, p. F4.

+ «Il y a quarante ans, Nègres blancs d'Amérique», Le Devoir, 26 avril 2008, p. F4.

+ "Voyage à Jonquière avec Charles Bukowski», Le Devoir, 3 mai 2008, p. F4.

+ «Le beau Danube noir», Le Devoir, 10 mai 2008, p. F4. 
+ «Bienvenue dans la Grosse Pomme», Le Devoir, 17 mai 2008, p. F4.

+ «L'écrivain de banlieue et le criminel de guerre», Le Devoir, 14 juin 2008, p. F4.

+ "L'affaire Morisset, ou le romancier malgré lui», Le Devoir, 21 juin 2008, p. F4.

+ «Plus dure est la chute», Le Devoir, 28 juin 2008, p. E7.

+ «Mourir pour nourrir», Le Devoir, 26 juillet 2008, p. E6.

+ "L'homme qui aimait rire», Le Devoir, 2 août 2008, p. E4.

+ «Les coureurs de froid», Le Devoir, 9 août 2008, p. E3.

+ «Le testament du mal», Le Devoir, 16 août 2008, p. E5.

+ «Le truc qui se lève toujours», Le Devoir, 23 août 2008, p. F4.

+ «Ça suffit, le bonheur!», Le Devoir, 30 août 2008, p. F4.

+ «Sous les galets, le bonheur», Le Devoir, 6 septembre 2008, p. F1.

+ "La machine à voyager dans le temps», Le Devoir, 13 septembre 2008, p. F4.

+ «Le Nord dans la peau, ou les 50 ans d'Agaguk», Le Devoir, 20 septembre 2008, p. F3.

+ «On a tiré sur la Lune», Le Devoir, 27 septembre 2008, p. F4.

+ «Un faux Canadien bien tranquille», Le Devoir, 11 octobre 2008, p. F4.

+ «Qu'est-ce qu'un auteur amérindien?», Le Devoir, 18 octobre 2008, p. F4.

+ «La guerre des langues», Le Devoir, $1^{\text {er }}$ novembre 2008, p. F6.

+ «La suspension de l'incrédulité....", Le Devoir, 8 novembre 2008, p. F4.

+ «La cavale canadienne de Jacques Mesrine», Le Devoir, 15 novembre 2008, p. F20.

+ "L'angoisse de la page imprimée», Le Devoir, 22 novembre 2008, p. F5.

+ «Une histoire de dupes», Le Devoir, 29 novembre 2008, p. F4.

+ "L'avidité du monde», Le Devoir, 6 décembre 2008, p. F4.

+ «Hommes du Nord», Le Devoir, 13 décembre 2008, p. F4.

+ «Les bâtards de Champlain», Le Devoir, 20 décembre 2008, p. F4.

+ «Agaguk en Floride, ou le Nord en plan», Le Devoir, 27 décembre 2008, p. E7.

+ «La forteresse», Le Devoir, 3 janvier 2009, p. E7.

+ «Un Pied-Noir sur la terre sacrée», Le Devoir, 10 janvier 2009, p. F4.

+ "L'année des aigles et des femmes», Le Devoir, 17 janvier 2009, p. F4.

+ «Les conséquences du sexe», Le Devoir, 31 janvier 2009, p. F4.

+ «Le col bleu devant John Updike (1932-2009)», Le Devoir, 7 février 2009, p. F4.

+ "Au pays du dos de la Grande Tortue», Le Devoir, 14 février 2009, p. F4.

+ «La faute à Hemingway», Le Devoir, 21 février 2009, p. F4.

+ "L'homme dont le métier était de se souvenir», Le Devoir, 7 mars 2009, p. F4.

+ «Des cœurs simples», Le Devoir, 14 mars 2009, p. F4.

+ «Ā feu et à sang», Le Devoir, 21 mars 2009, p. F4.

+ «Les richesses naturelles», Le Devoir, 28 mars 2009, p. F4.

+ «Entre la bonne et les fourneaux», Le Devoir, 4 avril 2009, p. F4.

+ «Lire John Berger est bon pour la santé», Le Devoir, 11 avril 2009, p. F4.

+ "Le dinosaure et les académies des lettres moulées», Le Devoir, 18 avril 2009, p. F4.

+ "L'invention du père», Le Devoir, 25 avril 2009, p. F4.

+ "Chili sans beaucoup de viande», Le Devoir, 2 mai 2009, p. F4.

+ «Le voyage en Mironie», Le Devoir, 9 mai 2009, p. F4.

+ "Parlons un peu du monstre...», Le Devoir, 16 mai 2009, p. F4.

+ "L'agence qui ne voulait rien savoir», Le Devoir, 23 mai 2009, p. F4.

+ «Petites bêtes de la forêt», Le Devoir, 30 mai 2009, p. F4. 
+ «La vente de garage», Le Devoir, 6 juin 2009, p. F4.

+ «Requiem pour la Romaine», Le Devoir, 11 juillet 2009, p. C5.

+ «Le malavenant», Le Devoir, 5 septembre 2009, p. F4.

+ "La bataille du Québec», Le Devoir, 12 septembre 2009, p. F4.

+ «La crise d'Octobre, une histoire de chiffre», Le Devoir, 19 septembre 2009, p. F7.

+ «Litanie de la dépossession», Le Devoir, 26 septembre 2009, p. F4.

+ «Profession: homme révolté», Le Devoir, 3 octobre 2009, p. H3.

+ «Les gros casques et José Acquelin», Le Devoir, 10 octobre 2009, p. F4.

+ «Un super-roman», Le Devoir, 17 octobre 2009, p. F4.

+ «Edipe, Updike, Nick et moi», Le Devoir, 24 octobre 2009, p. F4.

+ «De Rip van Winkle à Tom Cruise», Le Devoir, 31 octobre 2009, p. F4.

+ «Le choix du président», Le Devoir, 7 novembre 2009, p. F4.

+ «Le matou qui devint ministre», Le Devoir, 14 novembre 2009, p. F5.

+ «Docteur Lafond, je présume?», Le Devoir, 21 novembre 2009, p. F4.

+ «L'écrivain normal», Le Devoir, 28 novembre 2009, p. F4.

+ «Trois hommes, deux procès et un système», Le Devoir, 5 décembre 2009, p. F4.

+ «Les écrits des braves», Le Devoir, 12 décembre 2009, p. F4.

+ «Je rêve d'un Noël rouge», Le Devoir, 19 décembre 2009, p. F4.

+ «Carranza, ou les faces du pouvoir», Le Devoir, 24 décembre 2009, p. E4.

+ «Dix ans de cadeaux», Le Devoir, 31 décembre 2009, p. E7.

+ «Visa dans le noir, tua le vrai...», Le Devoir, 16 janvier 2010, p. F4.

+ «Pays sans tête», Le Devoir, 23 janvier 2010, p. F4.

+ "Les verts et les mûrs», Le Devoir, 30 janvier 2010, p. F4.

+ «Mourir aux larmes avec Crocodile Cook», Le Devoir, 6 février 2010, p. F4.

+ «Sylvia au bout du rouleau de la machine à écrire», Le Devoir, 13 février 2010, p. F4.

+ «La charge de Croc Gentil», Le Devoir, 20 février 2010, p. F4.

+ «Le Cabano des castors», Le Devoir, 27 février 2010, p. F4.

+ «Les gros bras», Le Devoir, 6 mars 2010, p. F4.

+ «Tombe les filles et fais des phrases», Le Devoir, 13 mars 2010, p. F4.

+ «Mourir en secret: la question de la torture», Le Devoir, 20 mars 2010, p. F4.

+ «La sainte paix», Le Devoir, 27 mars 2010, p. F4.

+ «Le vieux qui lisait des ouvrages marxistes», Le Devoir, 3 avril 2010, p. F4.

+ «Gaston Miron - l'avenir à rapailler», Le Devoir, 10 avril 2010, p. F1.

+ «Le vieil homme et la lagune», Le Devoir, 17 avril 2010, p. F4.

+ "L'Office de protection du lecteur», Le Devoir, 24 avril 2010, p. F4.

+ "Ceux qui vont mourir ou pas», Le Devoir, $1^{\mathrm{er}}$ mai 2010, p. F4.

+ «Le révolutionnaire qui inventa un écrivain», Le Devoir, 8 mai 2010, p. F4.

+ «Dublinescence, ou le dernier éditeur», Le Devoir, 15 mai 2010, p. F4.

+ «Le pari dickensien», Le Devoir, 22 mai 2010, p. F4.

+ «La cathédrale dans le jardin», Le Devoir, 29 mai 2010, p. F4.

+ «Une infinie tempête de beauté», Le Devoir, 5 juin 2010, p. F4.

+ «La vie d'artiste», Le Devoir, 19 juin 2010, p. F11.

+ «Hors saison», Le Devoir, 3 juillet 2010, p. E7.

+ «Debout après la folie», Le Devoir, 10 juillet 2010, p. E7.

+ «Retour à Natashquan», Le Devoir, 24 juillet 2010, p. E7. 
+ "Schefferville a vécu, Matimekosh s'accroche», Le Devoir, 29 juillet 2010, p. A1.

+ «La barricade», Le Devoir, 30 juillet 2010, p. A5.

+ «Hivernie mon pays», Le Devoir, 31 juillet 2010, p. E7.

+ «La vie aime la vie», Le Devoir, 7 août 2010, p. E7.

+ «Portraits de femmes», Le Devoir, 14 août 2010, p. E7.

+ "Je suis Finlandais», Le Devoir, 11 septembre 2010, p. F4.

+ "L'espion qui venait du bleu», Le Devoir, 25 septembre 2010, p. F4.

+ «Octobre 1970 - l'histoire d'une petite histoire», Le Devoir, 29 septembre 2010, p. A9.

+ «Libre opinion - pour sortir de la fiction», Le Devoir, 5 octobre 2010, p. A10.

+ «Salut Octobre», Le Devoir, 9 octobre 2010, p. F4.

+ «Les hippiphanies», Le Devoir, 16 octobre 2010, p. F4.

+ «Un nouveau, des nouvelles», Le Devoir, 20 octobre 2010, p. F5.

+ «Le mutilateur», Le Devoir, 23 octobre 2010, p. F4.

+ "Recherché mort et vif», Le Devoir, 6 novembre 2010, p. F4.

+ "Ceux qui ont raison», Le Devoir, 18 décembre 2010, p. F4.

+ «Il était une fois», Le Devoir, 24 décembre 2010, p. E7.

+ «La littérature en 2010 », Le Devoir, 31 décembre 2010, p. E7.

+ «Feuilles de Grass», Le Devoir, 8 janvier 2011, p. E9.

+ «Ceci était une pipe», Le Devoir, 29 janvier 2011, p. F4.

+ «Serge d'entre les morts», Le Devoir, 5 février 2011, p. F4.

+ «Mort un 2 juillet», Le Devoir, 12 février 2011, p. F4.

+ «Bienvenue dans le mirage», Le Devoir, 19 février 2011, p. F4.

+ "Quand la force fait l'union», Le Devoir, 26 février 2011, p. F4.

+ «Le piège à filles», Le Devoir, 5 mars 2011, p. F4.

+ «La légende du roi monté», Le Devoir, 19 mars 2011, p. F4.

+ «L'homme qui n'aimait pas les trotskistes», Le Devoir, 26 mars 2011, p. F4.

+ «Dans la tête de Sherman Alexie», Le Devoir, 2 avril 2011, p. F4.

+ «Pomme pourrie: une autre histoire de Guillaume Tell», Le Devoir, 9 avril 2011, p. F6.

+ «Leonard Cohen, un Québécois errant», Le Devoir, 16 avril 2011, p. F4.

+ «Naomi Fontaine, ou le regard neuf», Le Devoir, 23 avril 2011, p. F4.

+ «Cantate pour un temps de brume», Le Monde, 29 avril 2011, p. LIV4.

+ «Les invasions civilisées», Le Devoir, 30 avril 2011, p. F4.

+ «Un verre pour la route», Le Devoir, 7 mai 2011, p. F4.

+ «Un stylo pour le Labrador», Le Devoir, 21 mai 2011, p. F4.

+ «Les armes, les lettres et l'encensoir», Le Devoir, 28 mai 2011, p. F4.

+ «Hitler brûle-t-il?», Le Devoir, 11 juin 2011, p. F4.

+ «Ceux qui se battent», Le Devoir, 18 juin 2011, p. F4.

+ «Pour ne pas disparaître», Le Devoir, 2 juillet 2011, p. E7.

+ «Les 1211 jours du Roi du Coq Rôti, à Sherbrooke - le chant du cygne d'un interminable conflit», Le Devoir, 16 novembre 2011, p. B1.

+ «Un parvenu honnête et civilisé», Le Devoir, 7 janvier 2012, p. E7.

+ «Un homme et sa fiction», Le Devoir, 28 janvier 2012, p. F4.

+ «Voyage héroïque, aventure maudite», Le Devoir, 11 février 2012, p. F4.

+ «Dans la forêt et autour», Le Devoir, 25 février 2012, p. F4.

+ «Littérature américaine - l'attrape-queue», Le Devoir, 10 mars 2012, p. F4. 
+ «Du stade à l'asile», Le Devoir, 24 mars 2012, p. F4.

+ «Un air civilisé», Le Devoir, 14 avril 2012, p. F4.

+ «Une offre qu'ils ne pourront pas refuser», Le Devoir, 28 avril 2012, p. F4.

+ "Ceci n’est pas un récit de voyage», Le Devoir, 12 mai 2012, p. F4.

+ «Tropismes du Cône Sud», Le Devoir, 2 juin 2012, p. F4.

+ «Le pèlerinage sauvage», Le Devoir, 16 juin 2012, p. F4.

+ "Mortelle randonnée», Le Devoir, 30 juin 2012, p. E7.

+ «Le grand roman socialiste américain», Le Devoir, 14 juillet 2012, p. E7.

+ «L'anti-roman de plage», Le Devoir, 11 août 2012, p. E7.

+ «La route des baleines», Le Devoir, $1^{\text {er }}$ septembre 2012, p. F4.

+ "Alice au pays des merveilles», Le Devoir, 15 septembre 2012, p. F6.

+ "Le festival America - Les nouveaux guerriers», Le Devoir, 29 septembre 2012, p. F4.

+ «L'hallucination au pouvoir», Le Devoir, 13 octobre 2012, p. F4.

+ "Les chameaux de Zambra», Le Devoir, 27 octobre 2012, p. F4.

+ «Enrique Vila-Matas, une poétique de l'absence», Le Devoir, 10 novembre 2012, p. F4.

+ «Un salon du livre à Wendake», Le Devoir, 17 novembre 2012, p. F4.

+ «L'agenda rouge de Louise Erdrich», Le Devoir, 24 novembre 2012, p. F4.

+ «Rodolfo Walsh mort et vivant», Le Devoir, 8 décembre 2012, p. F4.

+ «Les temps superposés: chronique d'un non-liseur de poésie», Le Devoir, 12 janvier 2013, p. F4.

+ «Aventures séparatistes», Le Devoir, 26 janvier 2013, p. F5.

+ «La jungle est éternelle», Le Devoir, 23 février 2013, p. F4.

+ «La grosse classe», Le Devoir, 16 mars 2013, p. F4.

+ «Mon père parlait du Labrador», Le Devoir, 30 mars 2013, p. F4.

+ «Je partirai pour Québec», Le Devoir, 13 avril 2013, p. F4.

+ «Une journée américaine», Le Devoir, 27 avril 2013, p. F4.

+ «Ā propos de Doerr», Le Devoir, 11 mai 2013, p. F4.

+ «Stephen King devant JFK», Le Devoir, 25 mai 2013, p. F4.

+ «La lumière au bout du tunnel», Le Devoir, 8 juin 2013, p. F4.

+ «Himalaya de la rentrée», Le Devoir, 31 août 2013, p. F4.

+ «Dans l'ombre des grands hommes», Le Devoir, 14 septembre 2013, p. F4.

+ "L'argent parle, aux États-Unis comme au Québec», Le Devoir, 28 septembre 2013, p. F4.

+ «L'avant-dernier lecteur», Le Devoir, 16 novembre 2013, p. F7.

+ «Ā quoi ressemble un écrivain?», Le Devoir, 23 novembre 2013, p. F5.

+ «L'épreuve de la voix», Le Devoir, 7 décembre 2013, p. F5.

+ «L'année en romans», Le Devoir, 21 décembre 2013, p. E8.

+ «Le Québec d’Émilie Bordeleau», Le Devoir, 21 décembre 2013, p. E10.

+ «Il était des fois l'Amérique», Le Devoir, 11 janvier 2014, p. F5.

+ «Les champions poids lourd», Le Devoir, 25 janvier 2014, p. F4.

+ «Vanité de Ginsberg», Le Devoir, 8 février 2014, p. F4.

+ «La vie morale des écrivains», Le Devoir, 22 février 2014, p. F4.

+ «Littérature - Charles Bukowski, 20 ans plus tard», Le Devoir, 7 mars 2014, p. B2.

+ "Une grande dame du roman", Le Devoir, 8 mars 2014, p. F5.

+ "L'homme qui aimait les poètes», Le Devoir, 22 mars 2014, p. F4.

+ «Une histoire, des histoires», Le Devoir, 5 avril 2014, p. F6. 
+ «Un chasseur sachant écrire avec du chien», Le Devoir, 19 avril 2014, p. F5.

+ «Les batailles de Cocke», Le Devoir, 3 mai 2014, p. F5.

+ «Comment lire Proust au Texas», Le Devoir, 17 mai 2014, p. F5.

+ «Le fantôme qui parcourait le monde», Le Devoir, 31 mai 2014, p. F5.

+ "Mort d'un évêque progressiste», Le Devoir, 2 juin 2014, p. A4.

+ «Menu exotique», Le Devoir, 14 juin 2014, p. F5.

+ «Une nouvelle sortie pour Brooklyn», Le Devoir, 28 juin 2014, p. E7.

+ "David Treuer et "l'Ojibway of life"», Le Devoir, 12 juillet 2014, p. E7.

+ «La bande de Gaza», Le Devoir, 26 juillet 2014, p. E7.

+ "Traductions infidèles - La traduction n'est pas toujours une histoire d'amour», Le Devoir, 6 septembre 2014, p. F5.

+ «Traductions infidèles - De truchement à trucmuche, et de l'importance du nombre», Le Devoir, 20 septembre 2014, p. F4.

+ «Traductions infidèles - Tous des traducteurs», Le Devoir, 4 octobre 2014, p. F4.

+ «Traductions infidèles - La vie en double», Le Devoir, 18 octobre 2014, p. F5.

+ «Traductions infidèles - La littérature qui voyage», Le Devoir, $1^{\mathrm{er}}$ novembre 2014, p. F5.

+ «La dernière prise», Le Devoir, 15 novembre 2014, p. F8.

+ "La pêche aux choses envolées», Le Devoir, 29 novembre 2014, p. F5.

+ «Sur le Web», Le Devoir, 13 décembre 2014, p. F7.

\section{Articles parus dans des périodiques}

+ "Petite conversation de taverne. Entretien [avec Carolyn Cassady] (ou ce qui en tient lieu)», Voix et Images, vol. XIII, n³ 3, printemps 1988, p. 380-387.

+ «Portrait d'écrivain», Lettres québécoises, n 58, été 1990, p. 11-13.

+ "Ceux que vous lâchez», Liberté, vol. 33, nº 1, février 1991, p. 23-27.

+ «Dans le ventre du récit: lecture de Gérard Bessette», Voix et Images, vol. XX, n², hiver 1995, p. $440-450$.

+ «Réjean Ducharme, prix Athanase-David 1994 », Lettres québécoises, nº 77, printemps 1995, p. 7-10.

+ «Le scrap-book de la Contre-Amérique. Revoilà Yves Boisvert, en forme, solide comme un chêne, ou serait-ce plutôt un bouleau?», Lettres québécoises, n 87, automne 1997, p. 10-11.

+ «Un super-héros dépareillé», Liaison, n 95, janvier 1998, p. 19-21.

+ «De Mexico à Québec... le nouveau paradigme révolutionnaire», Possibles, vol. 25, nºs 3-4, automne 2001, p. 117-123.

+ «La sérénité du pêcheur à la ligne», L'Inconvénient, n 8, février 2002, p. 41-48.

+ «Une histoire émerveillée», Entre les lignes, vol. 1, n 4, été 2005, p. 24-25.

+ «Les armes et les lettres», Spirale, n² 206, janvier-février 2006, p. 39-40.

+ «La semence: Jacques Ferron et la crise d'Octobre», Possibles, vol. 29, ns 3-4, été-automne 2006, [pagination non disponible].

+ "L'écrivain de pays», L'Atelier du roman, no 47, septembre 2006, p. 23-31.

+ «Éloge du Survenant», L'Inconvénient, n² 28, février 2007, p. 79-89.

+ «Un gombo de roche gonzo: stratégies langagières chez Joyce et chez VLB», L'Action nationale, vol. XCVII, nºs 5-6, mai-juin 2007, p. 38-55.

+ «Lettre à un candidat à l'asile, à l'exil, au prix Nobel, etc.», Mobius, nº 118, automne 2008, p. 115-117. 
+ «D'orignaux et d'Indiens», Mœbius, n 124, février 2010, p. 109-113.

+ «Un bon génie: les héritiers de Don Quichotte I», Spirale, n²42, automne 2012, p. 7-9.

+ «La seconde vie de Pierre Laporte», L'Action nationale, vol. CII, n 9, novembre 2012, p. 104-117.

+ «Le bord de la falaise: les héritiers de Don Quichotte II», Spirale, n 243, hiver 2013, p. 7-9.

+ «Le conspirationnisme comme littérature de genre», L'Inconvénient, n 51, hiver 2013, p. 12.

+ «Le problème du moyen contexte: les héritiers de Don Quichotte III», Spirale, n 244 , printemps 2013, p. 7-9.

+ «Marco Polo contre les Tabarnacos», L'Inconvénient, n 53, été 2013, p. 63-70.

+ «Crier au loup: les héritiers de Don Quichotte IV», Spirale, n 245, été 2013, p. 7-9.

+ «Le grand homme de province», Les cahiers Victor-Lévy Beaulieu, n 4, 2014, p. 191-196.

\section{J. Traductions des œuvres de Louis Hamelin}

+ Cowboy. A novel [Cowboy], traduit en anglais par Jean Paul Murray, Toronto, Dundurn group, 2000, $309 \mathrm{p}$.

+ October 1970. A novel [La constellation du Lynx], traduit en anglais par Wayne Grady, Toronto, Arachnide, 2013, 614 p.

\section{K. Prix et distinctions}

+ Prix du Gouverneur général, La rage, 1990.

+ Finaliste: Prix Molson du roman, Ces spectres agités, 1991.

+ Sélection officielle: «Petite bibliothèque du parfait Montréalais», Ces spectres agités, 1992.

+ Finaliste: Prix littéraire du Journal de Montréal, Cowboy, 1993.

+ Finaliste: Prix des lectrices Elle Québec, Cowboy, 1993.

+ Finaliste: Prix France-Québec, Cowboy, 1993.

+ Finaliste: Grand Prix littéraire de la Ville de Montréal, Cowboy, 1993.

+ Finaliste: Prix du signet d'or, Betsi Larousse ou l'ineffable eccéité de la loutre, 1995.

+ Finaliste: Prix France-Québec, Betsi Larousse ou l'ineffable eccéité de la loutre, 1995.

+ Finaliste: Prix du Gouverneur général, Betsi Larousse ou l'ineffable eccéité de la loutre, 1995.

+ Finaliste: Prix Ringuet de l'Académie des lettres du Québec, Le soleil des gouffres, 1996.

+ Finaliste: Prix international de l'Union latine, Le soleil des gouffres, 1997.

+ Finaliste: Prix littéraire des collégiens, Le joueur de flûte, 2002.

+ Finaliste: Grand Prix littéraire de la Ville de Montréal, Sauvages, 2006.

+ Finaliste: Prix du Gouverneur général, Sauvages, 2006.

+ Finaliste: Grand Prix littéraire de la Ville de Montréal, La constellation du Lynx, 2010.

+ Grand Prix littéraire de la presse québécoise, La constellation du Lynx, 2011.

+ Prix littéraire des collégiens, La constellation du Lynx, 2011.

+ Prix des libraires du Québec, La constellation du Lynx, 2011.

+ Prix Ringuet de l'Académie des lettres du Québec, La constellation du Lynx, 2011.

+ Grand Prix du livre de la ville de Sherbrooke, La constellation du Lynx, 2012.

+ Amazon.ca, les 100 livres de 2013, October 1970. A novel, 2013.

+ Prix Giller, October 1970. A novel, 2013.

+ Canada Reads Top 10, October 1970. A novel, 2014.

+ Prix de la revue Études françaises, Fabrications. Essai sur la fiction et l'histoire, 2014. 


\section{RÉCEPTION CRITIQUE}

\section{A. Sur l'auteur et son ouvre en général}

1. Essai

+ OUELLET, François et François PARÉ, Louis Hamelin et ses doubles, Québec, Nota bene, 2008, 262 p.

\section{Chapitres de livre}

+ MARCOTTE, Gilles, "Générations», L'âge de la prose. Romans et récits québécois des années 80, Montréal/Rome, VLB éditeur/Bulzoni editore, 1992, p. 19-27.

+ DESMEULES, Georges et Christiane LAHAIE, Les classiques québécois, Québec, L'instant même, 1997, p. 84-87.

+ NOBLE, Peter, Frontières flottantes. Lieu et espace dans les cultures francophones du Canada, Amsterdam, Rodopi, 2001, p. 135-143.

+ OUELLET, François, Passer au rang de Père. Identité sociohistorique et littéraire au Québec, Québec, Nota bene, 2002, p. 72-79.

+ BIRON, Michel, «L'écrivain liminaire», Littératures mineures en langue majeure. Québec/ Wallonie-Bruxelles. Actes du colloque de Liège, 9-11 octobre 2001, New York/Montréal, P.I.E.Peter Lang/Presses de l'Université de Montréal, 2003, p. 57-67.

\section{Entrevue}

+ OUELLET, François, «Louis Hamelin en trois dimensions», Nuit blanche, n 53 , septembreoctobre-novembre 1993, p. 54.

\section{Mémoires et $t$ hès es}

+ SOULARD, Louis, La "génération x» dans le roman québécois actuel, mémoire de maîtrise, Montréal, Université McGill, 1995, 154 f.

+ DANDURAND, Thomas, Tradition et rupture dans La rage de Louis Hamelin, mémoire de maîtrise, Montréal, Université du Québec à Montréal, 1998, 147 f.

+ DUQUET, Geneviève, Du mimétisme au bouc émissaire. Présence du mythe de la violence fondatrice dans les romans Pelourinho et Ces spectres agités, mémoire de maîtrise, Québec, Université Laval, 1998, 80 f.

+ LECLERC, Céline, Le phénomène de l'assimilation dans La rage de Louis Hamelin, mémoire de maîtrise, Rimouski, Université du Québec à Rimouski, 1999, 123 f.

+ MATHIEU, Marie-Ėve, Les codes dans la littérature québécoise, mémoire de maîtrise, Montréal, Université du Québec à Montréal, 2000, 113 f.

+ LAPARÉ, Maude, L'inscription du littéraire dans Vamp de Christian Mistral et La rage de Louis Hamelin, mémoire de maîtrise, Montréal, Université de Montréal, 2001, 122 f.

+ MICHAUD, Marie-Andrée, Littérature, langue et société: la quête identitaire des Québécois depuis 1980, mémoire de maîtrise, Ottawa, Université d'Ottawa, 2002, 212 f.

+ VEILLET, Stéphanie, Rythme et coordination des extrêmes: pour une analyse de la métaphore animale et de l'affolement textuel dans La rage de Louis Hamelin, mémoire de maîtrise, Montréal, Université du Québec à Montréal, 2003, 91 f.

+ DANEAU, Robert, Enfers, suivi de Vie et mort du postmodernisme, thèse de doctorat, Sherbrooke, Université de Sherbrooke, 2004, 578 f.

+ MÉNARD, Valérie, L'influence de Réjean Ducharme chez les écrivains de la génération $X$, mémoire de maîtrise, Montréal, Université McGill, 2004, 129 f. 
+ DE LA ROCHE, David, Figures du marginal: Édouard Malarmé dans La rage de Louis Hamelin, suivi de Couteau et autres incantations, mémoire de maîtrise, Sherbrooke, Université de Sherbrooke, 2006, $104 \mathrm{f}$.

+ DESROCHERS, Julien, La rage de Louis Hamelin et le paradoxe sociocritique, mémoire de maîtrise, Québec, Université Laval, 2006, 104 f.

+ MÉNARD, Jean-Sébastien, Une certaine Amérique à lire: la beat generation et la littérature québécoise, thèse de doctorat, Montréal, Université McGill, 2007, $404 \mathrm{f}$.

+ LEPAGE, Élise, Géographie des confins. Espace et littérature chez Pierre Morency, Pierre Nepveu et Louis Hamelin, thèse de doctorat, Vancouver, University of British Columbia, 2010, $366 \mathrm{f}$.

+ MASSE, Marie-Claude, Le voyage engagé: pour une lecture politique du roman de la route actuel québécois, thèse de doctorat, Sherbrooke, Université de Sherbrooke, 2010, 250 f.

+ POISSANT, Maude, Ambivalences, suivi de La polyphonie dans La constellation du Lynx: Un dialogue explorant l'Histoire, mémoire de maîtrise, Québec, Université Laval, 2013, 135 f.

\section{Articles dans des quotidiens}

+ MARTEL, Réginald, «Louis Hamelin», La Presse, 6 novembre 1994, p. B1.

+ ÉMOND, Ariane, «Les ponts d'Ariane», Le Devoir, 3 décembre 1994, p. D5.

+ LACHANCE, Micheline, «Le biologiste du roman», L'Actualité, 15 novembre 1996, p.104.

+ CAYOUETTE, Pierre, «Quarante écrivains parleront d'amour», Le Devoir, 29 mars 1997, p. D2.

+ CHOUINARD, Marie-Andrée, "Le jardin du bout du monde», Le Devoir, 16 août 1997, p. D1.

+ CAYOUETTE, Pierre, «La rentrée de A à XYZ», Le Devoir, 7 septembre 1997, p. D2.

+ BORDELEAU, Francine, "Les ténèbres de l'Amérique», Le Devoir, 13 mars 1999, p. E25.

+ L'HEUREUX SAINTE-FOY, Claude, «Michaud, Sciortino et Hamelin», Le Devoir, 12 mars 2001, p. A6.

+ NADEAU, Jean-François, "Au bout des lettres», Le Devoir, 23 août 2003, p. F1.

+ RICHLER, Noah, «Libre opinion: Une comparaison odieuse», Le Devoir, 14 juin 2005, p. A6.

+ GIGUÈRE, Suzanne, «Un livre ne fait pas le printemps, mais... (sur un air connu)», Le Devoir, 28 janvier 2006, p. F3.

+ DOYON, Frédérique, «Le lecteur à l'orée de l'été», Le Devoir, 17 juin 2006, p. F1.

+ MONTPETIT, Caroline, «Le don de lire», Le Devoir, 16 décembre 2006, p. F1.

+ LAURIN, Danielle, «Écrire avec un gun», Le Devoir, 3 mars 2007, p. F3.

+ LAPIERRE, Michel, «VLB, le mangeur de géants», Le Devoir, 7 juillet 2007, p. E8.

+ BRÛLÉ, Michel, «Lettres: Sus au manichéisme», Le Devoir, 12 février 2008, p. A6.

+ LAURIN, Danielle, «De beaux lendemains», Le Devoir, 23 août 2008, p. F3.

+ DESMEULES, Christian, «Louis Hamelin sous le scalpel», Le Devoir, $1^{\text {er }}$ novembre 2008, p. F2.

+ HUSTON, Nancy, "Avec ou sans enfants», Le Devoir, 5 mai 2009, p. A6.

+ LAURIN, Danielle, "Romans québécois: valeurs sûres et découvertes», Le Devoir, 28 août 2010, p. F3.

+ DUFRESNE, Denis, «Sherbrookois d'adoption», La Tribune, 9 octobre 2010, p. W5.

+ LALONDE, Catherine, «Le Prix des libraires dévoile ses finalistes», Le Devoir, 26 janvier 2011, p. B8.

+ PORTER, Isabelle, «Prix des collégiens 2011 - Louis Hamelin remporte la grande élection littéraire des collégiens», Le Devoir, 16 avril 2011, p. A2.

+ LALONDE, Catherine, «Louis Hamelin remporte le 18e Prix des libraires du Québec», Le Devoir, 10 mai 2011, p. B8. 
+ CORNELLIER, Louis, «Échapper au réel pour le comprendre», Le Devoir, 14 janvier 2012, p. F1.

+ CORNELLIER, Louis, «Ā voir le jeudi 20 septembre - Entre la mémoire et le mal de vivre», Le Devoir, 15 septembre 2012, p. 32.

\section{Articles dans des périodiques}

+ MORENCY, Jean, «Louis Hamelin», Nuit blanche, n 51, mars-avril-mai 1993, p. 52-53.

+ AUDET, Noël, «Tendances actuelles du roman québécois», Lettres et cultures de langue française, $\mathrm{n}^{\circ} 20,1^{\text {er }}$ semestre 1994, p. 37-48.

+ SPEAR, Thomas C., «La plume bifide, le cœur québécois. L'usage de l'anglais chez les écrivains québécois francophones», Globe. Revue internationale d'études québécoises, vol. 4, nº 1, 2001, p. 71-91.

+ DUCHATEL, Annick, «Louis Hamelin. Simplicité volontaire», Entre les lignes, vol. 2, n 4, été 2006, p. 38-40.

+ LORD, Michel, «Marie José Thériault, Gilles Archambault, Louis Hamelin», Lettres québécoises, no 123, automne 2006, p. 35-36.

+ PARÉ, Yvon, «Danielle Laurin, Louis Hamelin, Alexandre Bourbaki», Lettres québécoises, nº 126, été 2007, p. 32-33.

+ BOISCLAIR, Isabelle et Lori SAINT-MARTIN, «Masculin/féminin chez les romanciers québécois contemporains: l'idée de différence entre maintien et renouvellement», Contemporary French and Francophone Studies, vol. 13, n 1, 2009, p. 45-54.

+ LABRÈCHE, Marie-Sissi, «Christian Mistral vu par Louis Hamelin», Lettres québécoises, nº 137, printemps 2010, p. 5-6.

+ BROCHU, André, "Louis Hamelin, Perrine Leblanc, William S. Messier», Lettres québécoises, no 142, été 2011, p. 19-20.

\section{Sur des ouvrages en particulier}

\section{a) La rage}

+ BIRON, Michel, «Professeurs d'espoir», Voix et Images, vol. 31, n 3, printemps 2006, p. 129-133.

+ BIRON, Michel, «Portrait de l'écrivain en autodidacte», @nalyses, vol. 2, n³ 3, automne 2007, en ligne: https://uottawa. scholarsportal. info/ojs/index. php/revue-analyses/article/view/671 (page consultée le 22 juillet 2015).

+ BOIVIN, Aurélien, «La rage ou le cri du cœur d'une génération perdue», Québec français, no 104, 1997, p. 85-88.

+ BOUTANTIN, Ėve, «Des écritures de la violence: Trou de mémoire, La rage et La petite fille qui aimait trop les allumettes», Études canadiennes/Canadian Studies, nº 61, décembre 2006, p. 25-37.

+ DESMEULES, Georges, «La rage», Nuit blanche, n³9, mars-avril-mai 1990, p. 20.

+ DESROCHERS, Julien, «De l'engagement collectif au repli narcissique. Représentation et influence de l'univers social dans quatre romans québécois des années 1980», Québec français, n 134, été 2004, p. 48-51.

+ DESROCHERS, Julien, «La rage de Louis Hamelin et la théorie du discours social de Marc Angenot. Une amorce de réflexion», Mélanie Carrier et Maude Poissant (dir.), Carrefour de lectures littéraires. États de la jeune recherche littéraire, Québec, Centre de recherche interuniversitaire sur la littérature et la culture québécoises (CRILCQ), coll. «Interlignes», 2006, p. 47-61. 
+ GRIGORUT, Constantin, «Un mythe voyageur et son ancrage intertextuel dans le roman La rage de Louis Hamelin", Études francophones, vol. 15, n 1, printemps 2000, p. 17-31.

+ HÉBERT, Pierre, «Expropriés d'aujourd'hui et d'autrefois», Voix et Images, vol. XV, n 3 , printemps 1990, p. 452-455.

+ LAMONTAGNE, André, "L'intertextualité territoriale: La rage de Louis Hamelin», Le roman québécois contemporain. La voix sous les mots, Montréal, Fides, 2004, p. 181-209.

+ LAMONTAGNE, André, "La rage», collectif, Dictionnaire des œuvres littéraires du Québec, t. VIII : 1986-1990, Montréal, Fides, 2011, p. 746-748.

+ LAMY, Jonathan, «Le chalet de l'américanité», Spirale, n 209, juillet-août 2006, p. 28-29.

+ LAPOINTE, Martine-Emmanuelle, «Le sens de l'histoire», Voix et Images, vol. XXXVI, n 2, hiver 2011, p. 141-145.

+ MARCOTTE, Gilles, «Trois romanciers d'une génération perdue: Sylvain Trudel, Christian Mistral, Louis Hamelin», La Licorne, n² 27, novembre 1993, p. 135-146.

+ MORENCY, Jean, "L'errance dans le roman québécois», Québec français, n 97, printemps 1995, p. 81-84.

+ PELLETIER, Jacques, «Le cri de rage d'une génération», Lettres québécoises, n 58, été 1990, p. 14-16.

+ PELLETIER, Jacques, «Préface. La rage d'une génération», Louis Hamelin, La rage, Montréal, TYPO, 1995 [1989], p. 7-12.

+ RESCH, Yannick, «L'écriture du terroir revisitée: La rage de Louis Hamelin», Romanica Silesiana, n 2, février 2007, p. 13-19.

+ SIMON, Sherry, «La rage», Spirale, n 98, été 1990, p. 11.

+ SMART, Patricia, "When "Next Episodes" Are No Longer an Option: Quebec Men's Writing in a Postfeminist, Postnationalist Age», Québec Studies, n 30, automne-hiver 2000, p. 28-43.

\section{b) Ces spectres agités}

+ BEAUDOIN, Nadia, «De la fuite à la quête dans plusieurs romans de jeunes Québécois de la dernière décennie", Québec français, n 115, automne 1999, p. 75-76.

+ BEAUDOIN, Réjean, «Le prix de la langue la plus ampoulée», Liberté, n 198, décembre 1991, p. 91-95.

+ BOIVIN, Aurélien et Cécile DUBÉ, «Les romanciers de la désespérance», Québec français, n 89, printemps 1993, p. 97-99.

+ BREUX, Sandra, «Ces spectres agités (Louis Hamelin, 1991)», Cahiers de géographie du Québec, vol. 52, nº 147, décembre 2008, p. 471-487.

+ CÔTÉ, Lucie, «Un portrait de l'écrivain... en vampire», La Presse, 10 février 1991, p. C3.

\section{c) Cowboy}

+ BIRON, Michel, «Du nord au sud», Voix et Images, vol. XVIII, n 3, printemps 1993, p. 610-614.

+ CÔTÉ, Lucie, «Louis Hamelin, un étranger au Far-west québécois», La Presse, 4 octobre 1992, p. B1.

+ OUELLET, François, "Portrait du héros "en jeune saint-je". La rage et Cowboy de Louis Hamelin", La problématique de l'identité dans la littérature francophone du Canada et d'ailleurs, Lucie Hotte (dir.), Ottawa, Le Nordir, 1994, p. 121-128.

+ RUGGERI, Paola, «Cowboy de Louis Hamelin. Le Far West québécois ou la redéfinition des frontières nordiques», Globe. Revue internationale d'études québécoises, 1998, vol. 1, nº 2, p. 9-27. 
+ SCOTT, Corrie, «Cowboys et Indiens. Masculinité, métissage et queeritude chez Tomson Highway et Louis Hamelin», temps zéro, n 7, décembre 2013, en ligne: http://tempszero. contemporain.info/document1108 (page consultée le 22 juillet 2015).

+ TREMBLAY, Emmanuelle, «Une identité frontalière. Altérité et désir métis chez Robert Lalonde et Louis Hamelin», Études françaises, 2005, vol. 41, nº 1, p. 107-124.

\section{d) Betsi Larousse ou l'ineffable éccéité de la loutre}

+ BEAUDOIN, Daniel-Louis, «Yeux fertiles. Louis Hamelin, Betsi Larousse ou l'ineffable eccéité de la loutre, XYZ éditeur, 1994, 271 p.», Mœbius, n 64, été 1995, p. 131-133.

+ LACHANCE, Micheline, «Les choix de l'été», L'Actualité, 15 juin 1995, p. 82.

+ LECLERC, Céline, «Une rencontre entre nature et culture (Betsi Larousse ou l'ineffable éccéité de la loutre, de Louis Hamelin)», Tangence, n 49, décembre 1995, p. 119-120.

+ LEQUIN, Lucie, "Sur la route, vues de l'intérieur», Voix et Images, vol. XX, n 3, printemps 1995, p. 717-720.

+ SOURIEAU, Marie-Agnès, "Books Review: Betsi Larousse ou l'ineffable eccéité de la loutre», The French Review, vol. 70, n² 2, hiver 1996, p. 349-350.

+ VIGNES, Sylvie, «Explorations et inventions des mondes dans Betsi Larousse de Louis Hamelin», Études canadiennes/Canadian Studies, n 67, décembre 2009, p. 29-41.

\section{e) Le soleil des gouffres}

+ CAYOUETTE, Pierre, "Louis Hamelin: l'Amérique intérieure», Le Devoir, 9 novembre 1996, p. D3.

+ COTÉ, Jean-François et Sophie BÉLANGER, «La nord-américanité en roman: Le soleil des gouffres de Louis Hamelin», Voix et Images, vol. XXV, n 3, printemps 2000, p. 525-540.

+ ROY, Mario, "Mirages», La Presse, 3 novembre 1996, p. B1.

+ SURPRENANT, Jean-Claude, «Le soleil de Louis Hamelin», Le Droit, 16 novembre 1996, p. A12.

\section{f) Le voyage en pot}

+ DESAUTELS, Vincent, «Écriture libre sur commande», Le Devoir, 8 janvier 2000, p. D3.

\section{g) Le joueur de flûte}

+ BOURGAULT-CÔTÉ, Guillaume, «L'art du direct», Le Soleil, 11 novembre 2001, p. B3.

+ BROCHU, André, «La brigande, le mourant et le joueur de flûte», Lettres québécoises, nº 106, été 2002, p. 13-14.

+ CHARTRAND, Robert, «Roman québécois, À l'Ouest, là-bas, les origines...», Le Devoir, 10 novembre 2001, p. D5.

+ FERLAND, Pierre-Paul, «"Les voyages forment la jeunesse": mythe américain et rite initiatique dans le roman de la route québécois contemporain", Québec français, n² 164, hiver 2012, p. 38-41.

+ LEPAGE, Élise, «De l'utopie vers l'Éden: Le joueur de flûte de Louis Hamelin», Voix plurielles, 2009, vol. 6, nº 1, en ligne: http://brock.scholarsportal.info/journals/voixplurielles/article/ view/172 (page consultée le 22 juillet 2015). 


\section{h) L'humain isolé}

+ CARPENTIER, André, «L'humain isolé (review)», University of Toronto Quarterly, vol. 79, n 1 , hiver 2010, p. 118-121.

+ NADEAU, Jean-François, «En aparté - Question», Le Devoir, 23 septembre 2006, p. F2.

+ NAREAU, Michel, «Louis Hamelin et l'écriture», Nuit blanche, nº 107, été 2007, p. 12-13.

\section{i) Sauvages}

+ DESMEULES, Christian, «Louis Hamelin, prédateur malgré lui», Le Devoir, 11 février 2006, p. F1.

+ DESMEULES, Christian, "Quelques choix pour la saison», Le Devoir, 17 juin 2006, p. F4.

+ FORTIN, Marie-Claude, «L'homme des cavernes», La Presse, 2 juillet 2006, p. A1.

+ LAURIN, Danielle, «Fini la rage, Louis Hamelin propose Sauvages», Le Droit, 11 mars 2006, p. A20.

\section{j) La constellation du Lynx}

+ BERGERON, Carl, «Une œuvre fondatrice: La constellation du Lynx», L'Action nationale, vol. 100, nºs 9-10, novembre-décembre 2010, p. 35-40.

+ COLEMAN, Patrick, «That Doleful October, 40 Years Later - 2: for Louis Hamelin, Quebec Is Characterized by its Flatness", Inroads, automne-été 2011, n 29, p. 130.

+ FESSOU, Didier, «Du vrai autant que du faux», Le Soleil, 3 octobre 2010, p. 40.

+ FOURNIER, Louis, «La vraie histoire de la crise d'Octobre», Le Devoir, 30 septembre 2010, p. A9.

+ GENDRON, Guy, «Réplique à Louis Hamelin - Nos reportages n'accréditent pas votre thèse», Le Devoir, 30 septembre 2010, p. A9.

+ GUY, Chantal, «Octobre, le roman», La Presse, 2 octobre 2010, p. A20.

+ GUY, Chantal, «Du complot comme genre littéraire», La Presse, 8 janvier 2013, p. A2.

+ LAPIERRE, Michel, «Louis Hamelin et les étoiles d'Octobre», Le Devoir, 25 septembre 2010, p. F1.

+ LAPIERRE, Michel, «Octobre 70 et le procès du juridisme», Le Devoir, 9 octobre 2010, p. F6.

+ LAPLANTE, Laurent, «La constellation du Lynx: L'ample Octobre de Louis Hamelin», Nuit blanche, $\mathrm{n}^{\circ}$ 121, hiver 2011, p. 42-44.

+ LAPOINTE, Josée, «La police a laissé perdurer la crise», La Tribune, 2 septembre 2014, p. 22.

+ LESSARD, Valérie, «Hamelin creuse la crise d'Octobre», Le Devoir, 25 septembre 2010, p. A4.

+ MERCIER, Noémi, «La bombe Hamelin», L'Actualité, 15 octobre 2010, p. 20.

+ ROBITAILLE, Antoine, «Les théories du complot», Le Devoir, 30 septembre 2010, p. A1.

+ RUEL, Hélène, "La culture, c'est fait pour s'interroger», La Nouvelle Union, 26 octobre 2014, p. 8.

+ TREMBLAY, Odile, «Ā chacun son Octobre», Le Devoir, 2 octobre 2010, p. E2.

\section{k) Fabrications. Essai sur la fiction et l'histoire}

+ DUPONT, Éric, «La main invisible», L'Actualité, 15 novembre 2014, p. 69.

+ LIVERNOIS, Jonathan, «Fabriquer l'histoire», Le Devoir, 27 août 2014, p. B8. 